\title{
Patent Foramen Ovale, Factor V Leiden Heterozygosity and IgA Nephropathy: A Gase Report
}

\section{JCR}

Fatima M. Ezzeddine' ${ }^{1}$, Marwa R. Al-Badri ${ }^{2}$, Ahmad A. El Alayli ${ }^{3}$, Sami T. Azar ${ }^{2}$

Faculty of Medicine, American University of Beirut-Medical Center ${ }^{1,3}$; Department of

Internal Medicine, Division of Endocrinology, American University of Beirut-Medical

Center ${ }^{2}$, Beirut, Lebanon.

\section{Abstract:}

Background: People with patent foramen ovale are prone to develop strokes due to paradoxical embolization. Determination of association of coagulation disorders with patent foramen ovale is important to determine prognosis and avoid thromboembolic complications. Case Report: Our patient is a 25 year old Lebanese man with history of patent foramen ovale diagnosed at age of 1 year. He presented with severe epistaxis, hemoptysis and melena (INR>6). A kidney biopsy done a year ago showed that the patient has IgA nephropathy. Hypercoagulability workup done on this admission revealed that the patient also has factor $V$ Leiden heterozygosity. Consequently, this patient is at an increased risk of thromboembolic events and developed a stroke at seventeen years of age. Conclusion: This case underlines the importance of special management of patients with patent foramen ovale along with multiple comorbidities resulting in a hypercoagulable state such as IgA nephropathy and factor $V$ Leiden heterozygosity. Hence, further future studies should be conducted to identify the optimal management of these patients.

Key words: Foramen Ovale, Factor V, Stroke, Melena, Epistaxis, Hemoptysis.

\section{Introduction}

Factor $V$ Leiden thrombophilia is characterized by an increased risk for venous thromboembolism. The clinical expression of factor $V$ thrombophilia is influenced by the number of factor $V$ Leiden alleles and the presence of acquired hypercoagulable disorders such as $\lg A$ nephropathy [1]. $\lg A$ nephropathy is the most common form of glomerulonephritis, a principal cause of ESRD (end stage renal disease) worldwide. Kidneys of people with $\lg A$ nephropathy show deposits of lgA-containing immune complexes with proliferation of the glomerular mesangium and typical clinical features include onset before age 40 with hematuria, proteinuria and episodes of gross hematuria following mucosal infections [2].

$\lg$ A nephrotic syndrome is associated with blood hypercoagulability and a risk of thromboembolism additive to the one due to the inherited thrombophilia. Consequently, a special approach for management should be considered in patients with patent foramen ovale. Herein, we

\section{Corresponding Author: Dr. Sami T. Azar}

Email: sazar@aub.edu.lb

Received: March 3, 2015 | Accepted: May 1,2015 | Published Online: June 5, 2015

This is an Open Access article distributed under the terms of the Creative Commons Attribution License (creativecommons.org/licenses/by/3.0)

Conflict of interest: None declared | Source of funding: Nil | DOl: http://dx.doi.org/10.17659/01.2015.0060 
present the case of a patient with a patent foramen ovale, factor $\mathrm{V}$ Leiden heterozygosity and $\lg \mathrm{A}$ nephropathy.

\section{Case Report}

A 25 year old Lebanese male patient presented to the American University of Beirut Medical Center (AUBMC) in July 2014 for severe epistaxis (INR>6) associated with hemoptysis and melena. He reports no previous episodes of epistaxis. In the emergency department, he received four units of fresh frozen plasma products and $10 \mathrm{mg}$ vitamin $\mathrm{K}$. The patient has a patent foramen ovale, pulmonary valve repair done when he was one year old and IgA nephropathy diagnosed one year ago. He also had a stroke when he was seventeen years old with no residual neurological deficits. Prior to admission, he was taking: irbesartan+hydrochlorothiazide (150 mg, 1 tablet daily), bisoprolol $(2.5 \mathrm{mg}, 1$ tablet daily) and warfarin ( $4 \mathrm{mg}, 1 / 2$ tablet daily). He was regularly monitoring his INR monthly except for last month when INR monitoring was not done.

A carotid duplex performed in July, 2014, revealed minimal plaque deposition at the carotid bifurcation, bilaterally and a patent vertebral artery with anterograde flow, bilaterally. MRI of the brain, revealed evidence of mild atrophy and gliosis involving left post-central gyrus and posterior aspect of left insula, sequelae of old small left MCA infarct. Echocardiography, revealed flattening of the interventricular septum in systole and diastole secondary to right ventricular pressure and volume overload, mild right atrial dilation, pulmonary valve repair, severe pulmonary regurgitation (grade 4), mild pulmonary stenosis and moderate tricuspid valve regurgitation (grade 2). A hypercoagulability work-up was done and the patient was found to have factor $V$ Leiden heterozygosity. In addition, the patient has $\lg \mathrm{A}$ nephropathy diagnosed by a CT guided kidney biopsy performed in May, 2014. He clinically presented at that time with hematuria and proteinuria which were documented on laboratory testing.

After reviewing the literature, our case seems to be the first case report of a patient with patent foramen ovale, factor $V$ Leiden heterozygosity and $\lg A$ nephropathy which requires a different approach for management and prophylactic anticoagulation.

\section{Discussion}

More than $40 \%$ of ischemic strokes that occur in people less than 55 years are cryptogenic of whom $50 \%$ are found to have a patent foramen ovale. Coagulation abnormalities promote paradoxical emboli in these patients. Factor $V$ Leiden mutation is associated with three to six fold increase in risks for primary and recurrent venous thromboembolic events especially in patients without transient risk factors such as surgery and trauma [3].

A patent foramen ovale is a remnant of fetal circulation and permits microemboli to escape the pulmonary filter into the intracranial circulation causing stroke [4]. In addition, studies in ischemic stroke patients have showed that factor $V$ Leiden gene mutation is more frequent in patients with patent foramen ovale than those without [4]. Patients with cryptogenic strokes and patent foramen ovale have increased atrial vulnerability and can have greater potential for paroxysmal atrial fibrillation and thrombus formation. This explains why the patient was placed on warfarin after developing the stroke.

The thromboembolic events are increased in nephrotic patients with hypoalbuminemia being the most significant independent predictor factor of thrombotic risk [5]. This hypoalbuminemia causes urinary losses of anticoagulants and increased liver synthesis of procoagulants [6]. This is considered an additional risk to his inherited thrombophilia and further supports the use of warfarin to anticoagulate prophylactically. 
The presence of both factor $V$ Leiden heterozygosity and IgA nephropathy is what makes this patient with patent foramen ovale critical noting that no studies have shown an involvement of factor $V$ Leiden heterozygosity in the pathogenesis of IgA nephropathy or a link between these two disorders. Literature review showed only recent studies investigating the association of mild factor $V$ deficiency rather than factor $V$ heterozygosity with $\lg A$ nephropathy [7].

\section{Conclusion}

The occurrence of strokes at a young age is uncommon. However, paradoxical embolization can explain it and this risk is increased by the presence of factor $V$ Leiden heterozygosity and IgA nephropathy and should be addressed in the management. Whether simple anticoagulation is enough or need for correction of the patent foramen ovale is a question to be answered in future studies.

\section{References}

1. Kujovich, J. Factor V Leiden Thrombophilia. Gene Reviews. Portland, Oregon 2010.
2. Gharavi A. IgA nephropathy, the most common cause of glomerulonephritis, is linked to 6q2223. Nature Genetics. 2000;26(3):354-357.

3. Price $D$, Ridker P. Factor $V$ Leiden mutation and the risks for thromboembolic disease: $A$ clinical perspective. Annals of Internal Medicine. 1997; 1 27(10):895-903.

4. Desai A, Fuller C, Jesurum J, Reisman M. Patent Foramen Ovale and Cerebrovascular Disease. Nature Clinical Practice Cardiovascular Medicine. 2006;3(8):446-455.

5. Gigante A, Barbano B, Sardo L, Martina P, Gasperini M, Labbadia R, Cianci R. Hypercoagulability and nephrotic syndrome. Curr Vascular Pharmacology. 2014;12(3):512517.

6. Kanfer A. Coagulation factors in nephrotic syndrome. Am J Nephrology. 1990;10(1):6368.

7. Imai H, Kodama T, Yasuda T, Wakui H, Komatsuda A, Miura $A B$, Nakamoto Y. IgA nephropathy associated with mild type-coagulation factor $\checkmark$ deficiency in father and son. Clin Nephrol. $1995 ; 44(2): 133-134$. 